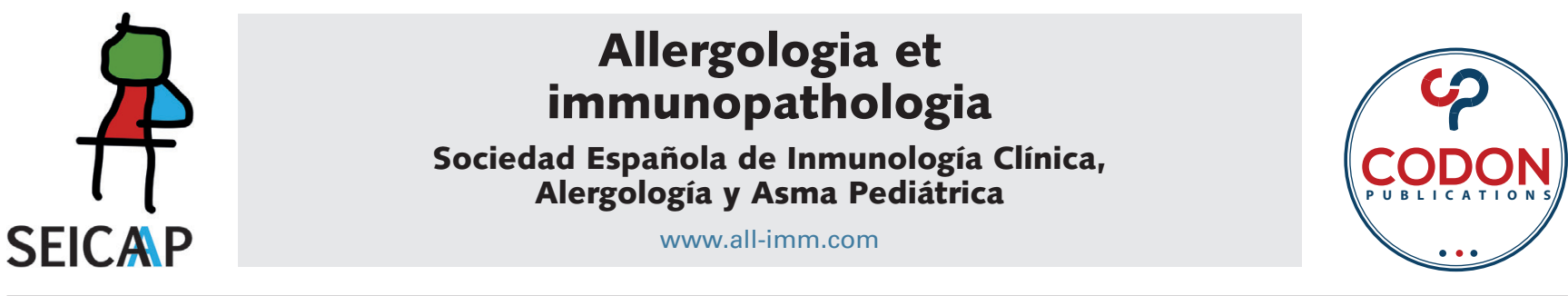

ORIGINAL ARTICLE

\title{
MiR-224 ameliorates inflammation and symptoms in mouse model of allergic rhinitis by targeting CDK9
}

\author{
Sang Wang ${ }^{\dagger}$, Lei Wang ${ }^{\dagger}$, Hua Hu*, Pin Dong*
}

Division of ENT, Key Laboratory of Head and Neck, Shanghai General Hospital of Nanjing Medical University, Shanghai, PR China

Received 23 May 2021; Accepted 6 October 2021

Available online 5 November 2021

\section{KEYWORDS \\ rhinitis; \\ allergic; \\ microRNAs; \\ cyclin-dependent \\ kinase 9; \\ genetic therapy}

\begin{abstract}
Objectives: To explore the regulatory effects of microRNA (miRNA)-224 and its potential target gene, cyclin dependent kinase 9 (CDK9), in the pathological process of allergic rhinitis (AR). Methods: To investigate the role of miR-224 and CDK9, it was screened by bioinformatics prediction software and verified by dual-luciferase reporter assay. The mouse model of AR was established by ovalbumin (OVA). The animal models were intervened with miR-224 agomir, negative control agomir, and saline respectively. The symptoms of sneezing and nasal rubbing were recorded. The expressions of miR224, CDK9, and cytokines in the nasal mucosa of different groups were analyzed by rt-PCR or western blotting. Enzyme-linked immunoassay (ELISA) was used to evaluate the levels of IgE and Histamine (HA) in the serum. The infiltration of inflammatory cells in the nasal mucosa was studied by immunohistochemistry. The expression and distribution of CDK9 in the nasal mucosa of mice were revealed by immunofluorescence.

Results: In the nasal mucosa of the animal models, the level of miR-224 was downregulated, while that of CDK9 was upregulated. The upregulation of miR-224 by miR-224 agomir reduced the frequencies of nasal rubbing and sneezing, the expression of CDK9, the levels of cytokines, and the concentrations of IgE and HA. Moreover, miR-224 appeared to attenuate the infiltration of inflammatory cells and hypersecretion of glands in the nasal mucosa. The expression of CDK9, which was distributed under the mucosa, especially in the submucosa interstitial tissue, was significantly reduced.

Conclusion: MiR-224 affected the pathogenesis of AR by targeting CDK9. It proves that miR-224 could be a novel potential therapeutic target for AR.

(c) 2021 Codon Publications. Published by Codon Publications.
\end{abstract}

*Corresponding authors: Pin Dong, PhD, Division of ENT, Key Laboratory of Head and Neck, Shanghai General Hospital of Nanjing Medical University, Shanghai 200080, PR China. Email address: dpshgh@yeah.net; Hua Hu, PhD, Division of ENT, Key Laboratory of Head and Neck, Shanghai General Hospital of Nanjing Medical University, Shanghai 200080, PR China. Email address: hua.hu@outlook.com

tThe first two authors contributed equally to this paper. 


\section{Introduction}

Allergic rhinitis (AR) is a complex allergic disease of rhinology with multigene involvement. One of the most common allergic diseases in the world, the prevalence of AR worldwide is $10 \%$ to $40 \%$, and in China it has exceeded $17 \%{ }^{1}$ with a significant upward trend in recent years. AR seriously affects patients' quality of life, and at the same time adds a huge medical and socio-economic burden on them. The pathogenesis of AR is complex. A variety of immunoreactive and pro-inflammatory cells and cytokines are thought to be involved in it at present, ${ }^{2}$ but no satisfactory treatment for $A R$ is available. The developments in the the molecular pathological mechanisms reveal that there are differential expressions of multiple gene sequences in the pathogenesis of AR, involving multiple ribonucleic acids (RNA) such as long-stranded non-coding RNA (IncRNA) and microRNA (miRNA). MiRNAs are a class of small molecule RNAs which was discovered in recent years that affect and regulate cell growth, differentiation, and function. The MiRNAs play an important role in the post-transcriptional regulation of protein-coding gene expression, thereby affecting a range of physiological and pathological processes in the organism. AR is a disease associated with multiple genes, and thereby miRNAs may participate in its development. A previous study showed that compared to the control group, a total of 421 miRNAs were differentially expressed in the nasal mucosa of AR patients, and nine miRNAs in AR group had significant differences with twofold change. A previous study showed that compared to the control group, 421 miRNAs were differentially expressed in the nasal mucosa of AR patients, and 9 miRNAs in AR group had significant differences with twofold change. Of these 9 miRNAs, 7 (miR-498, miR187, miR-874, miR-143, miR-886-3p, miR-224, and miR-767-5p) decreased in expression and 2 (miR-7 and miRPlus-E1194) increased. ${ }^{3}$ MiR-224 is significantly downexpressed in the nasal mucosa of AR patients, suggesting that it may be involved in the regulatory network of genes associated with allergic inflammation, but its mechanism requires further study. In the present study, the possible target genes of miR-224 were predicted by miRNA target gene prediction software and database respectively. The intersection of at least two target gene prediction software was taken as a result, and cell cycle protein-dependent kinase 9 (CDK9) was finally screened as one of the potential target genes of miR-224, and its role in AR was explored.

CDK9 is a member of the serine/threonine kinase family, which is essential in the whole cell cycle and apoptosis. CDK9 is involved in inflammatory response, ${ }^{4}$ viral infection, ${ }^{5}$ and tumor cell regulation. ${ }^{6}$ Th2 lymphocyte cytokines, mast cell, and endothelial cell related cytokines, including IL-4, IL-5, IL-6, IL-13, etc. modulate the immune reaction in AR. CDK9 interacts with the receptor of IL- 6 family of cytokines, gp130, and affects the inflammatory reaction. ${ }^{7}$ Additionally, the differentiation and apoptosis of Treg cells are both associated with the pathologic process of AR. 8 Study has shown that CDK9 inhibition increases the regulatory $\mathrm{T}$ cell population, induces apoptosis in leukocyte subsets PBMCs, and modulates the immune response. ${ }^{9}$ Therefore, a change the expression of CDK9 will likely benefit the treatment of human diseases, ${ }^{10,11}$ including allergic diseases. CDK9 has been extensively studied in the field of tumor therapy, and its regulatory mechanisms are still being explored. CDK9 is closely related to the release of various cytokines and the regulation of RNA. Based on the fact that $A R$ is a multi-gene disease with complex pathogenesis, the mechanism of CDK9's role in it needs to be further explored.

In this experiment, the potential target gene CDK9 of miR-224 was predicted, screened, ad validated. Furthermore, we used miR-224 agomir to intervene the AR mouse model to examine the regulatory function of miR224 on the target gene, and to explore the role of miR-224 and CDK9 in the pathogenesis of AR, which will provide a theoretical basis for future viable gene therapy for AR.

\section{Materials and methods}

\section{AR animal model}

All animal experiment procedures were carried out according to the Animal Testing Guideline of the Animal Laboratory Centre of our affiliation. C57 mice (male, 8-weeks old) were provided by the Animal Laboratory Centre of our affiliation. The mice were randomly divided into four groups of eight mice: (1) AR group; (2) AR+agomiR224 (miR224 agomir) intervention group; (3) AR+NC-miRNA (negative control miRNA agomir) control group; (4) saline control group. AgomiR224 and NC-miRNA were synthesized by Shanghai Jima Bio. The mice of the first three groups were injected intraperitoneally with OVA $(40 \mu \mathrm{g} / \mathrm{kg}$, Solarbio, Beijing, China) and aluminum hydroxide gel (40 mg/kg, Sigma Aldrich, USA) on day 1 , day 7 , day 14 , and day 21 . The saline control group was injected intraperitoneally with saline at the same time. After the last abdominal sensitization, nasal drop (morning) was administered in the moring to the latter three groups of mice as the process of intervention using agomiR224 (20 $\mu \mathrm{L}, 1 \mu \mathrm{g} / \mu \mathrm{L})$, NC-miRNA $(20 \mu \mathrm{L}$, $1 \mu \mathrm{g} / \mu \mathrm{L})$, and saline $(20 \mu \mathrm{L})$, respectively. Subsequently, the AR groups of mice were challenged to sensitize by the continuous intranasal drop of OVA $(20 \mu \mathrm{L}, 25 \mathrm{mg} / \mathrm{mL})$ for three days (afternoon). The intervention and OVA challenged processes were then repeated thrice more (4 times in total). There was a six hour interval between intervention and challenge (Figure 1). After the last nasal challenge, the numbers of sneezing and nasal rubbing of the mice were recorded during a 10 minute intervel period.

The mice were anesthetized by intraperitoneal injection of sodium pentobarbital $(40 \mathrm{mg} / \mathrm{kg})$. Fresh blood from the tail of each mouse was divided into two tubes, and the blood samples were centrifuged and stored at $-20^{\circ} \mathrm{C}$. After execution of the mice, the nasal cavity of the mice was quickly dissected to obtain the nasal mucosal tissue, which was immediately transferred to $-70^{\circ} \mathrm{C}$ liquid nitrogen for storage. In addition, nasal tissue was fixed in $10 \%$ formaldehyde for histopathological testing.

\section{Bioinformatics analysis}

Several miRNA target gene prediction software and databases were used, including TargetScan, miRanda, PITA, picTar, miRwalk, miRTarBase, etc. The predicted target 


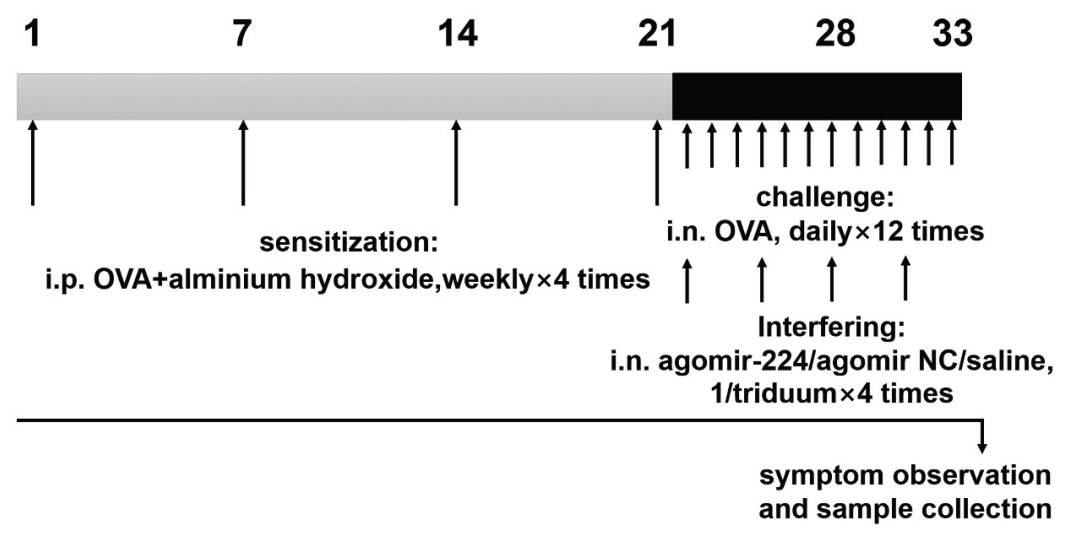

Figure 1 The process of animal experimentation. Mice were sensitized by intraperitoneal injection of OVA and aluminum hydroxide gel solution on days 1, 7, 14 and 21. On days 22 to 33, the mice were nasally stimulated continuously with OVA, and nasal drops (agomiR-224, NC-miRNA, or saline) were administered to the mice. After the last nasal challenge, the numbers of sneezing and nasal rubbing of the mice were recorded, and nasal mucosal tissue and serum were collected.

genes of miR-224 were predicted and exported separately through online service sites. After the localization run, the intersections were taken, and the intersections of at least two target gene prediction software were taken as the results.

\section{Cell culture and miRNA transfection}

Human nasal mucosal epithelial cells (HNECs) were collected from non-chronic nasal-sinusitis patients undergoing nasal endoscopic surgery by gentle brushing in, a method described by $\mathrm{Hu}$ et al. ${ }^{12}$ (approved by the ethics committee of our affiliation). HNECs were maintained with PneumaCultTM-Ex Plus medium (Stem Cell Technologies, Vancouver, Canada) in a cell incubator at $37^{\circ} \mathrm{C}$, with $5 \%$ $\mathrm{CO}^{2}$. The transfection of each group was performed according to the instructions of Lipofectamine 2000 (Invitrogen Co., USA).

\section{Dual-luciferase reporter assay}

Dual-luciferase reporter assay was performed to verify the relationship between miR-224 and the target gene CDK9 based on the results of bioinformatics analysis. The wild type CDK9 3'UTR segment (Genbank accession No. NM_001261) was amplified and cloned into the pGL3-Promoter vector (Promega Co., USA). The mutant CDK9 3'-UTR binding site sequence was determined based on hsa-miR-224-5p and CDK9 3'-UTR binding site, which was inserted into the vector pGL3-Promoter to construct pGL3-Promoter-mutCDK9. HNECs were co-transfected with agomiR224 or NC-miRNA and 3'UTR CDK9 (with wild type or mutant miR-224 binding sites) for 48 hours by Lipofectamine 2000, and then assayed with the dual luciferase reporter assay system (Promega, Madison, WI, USA).

\section{Real-time PCR}

Total RNA was extracted from mouse nasal mucosal tissue according to the Trizol reagent instructions (ABI-7300, Thermo Fisher, USA). After eliminating the DNA from the total RNA, the first strand of CDNA was synthesized using a reverse transcription kit (Fermentas, Canada). The SYBR Green PCR kit (Thermo Fisher, USA) and the ABI-7300 realtime detector were then used for real-time quantitative PCR detection. The sequences of primers specific to the genes tested are listed in Table 1 . The reaction procedure was as follows: PCR amplification: $95^{\circ} \mathrm{C}, 10 \mathrm{~min} ;\left(95^{\circ} \mathrm{C}, 15\right.$ $\left.\mathrm{Sec} ; 60^{\circ} \mathrm{C}, 45 \mathrm{Sec}\right) \times 40$ cycles; melting curve: $95^{\circ} \mathrm{C}$, $15 \mathrm{Sec}$; $60^{\circ} \mathrm{C}$, $1 \mathrm{~min} ; 95^{\circ} \mathrm{C}$, $15 \mathrm{Sec} ; 60^{\circ} \mathrm{C}$, $15 \mathrm{Sec}$. Data analysis was performed by ABI Prism 7300 SDS software.

\section{Western blotting}

Total tissue protein was extracted by RIPA tissue cell rapid lysis fluid (JRDUN Biotechnology, Shanghai). Protein concentration was quantified by BCA protein quantification kit (Thermo Fisher, USA). An aliquot of $50 \mu \mathrm{g}$ protein was added to a $10 \%$ polyacrylamide gel. After electrophoresis, the sample was transferred to a polyvinylidene fluoride (PVDF) membrane, and closed with 5\% skimmed milk powder at $4^{\circ} \mathrm{C}$ overnight. The membrane was then left at room

Table 1 The primer sequences used in RT-PCR.

\begin{tabular}{lll}
\hline Genes & & Pimers sequences $\left(5^{\prime}\right.$ to 3' $\left.^{\prime}\right)$ \\
\hline MiR-224 & Forward & CGCGCGTAAGTCACTAGTGGT \\
& Reverse & AGTGCAGGGTCCGAGGTAT \\
CDK9 & Forward & AGGTGGCTCTGAAGAAAGTG \\
& Reverse & AGCGGTTATACGGTGAGG \\
IL-4 & Forward & GTTGTCATCCTGCTCTTC \\
& Reverse & GTTTGGCACATCCATCTC \\
IL-6 & Forward & GAGGATACCACTCCCAACAGACC \\
& Reverse & AAGTGCATCATCGTTGTTCATACA \\
IL-18 & Forward & CGTGTTCCAGGACACAACAAG \\
& Reverse & TACAGGCGAGGTCATCACAAG \\
GAPDH & Forward & CTGCCCAGAACATCATCC \\
& Reverse & CTCAGATGCCTGCTTCAC \\
U6 & Forward & GCTTCGGCAGCAC \\
& Reverse & GGAACGCTTCACG \\
\hline
\end{tabular}


temperature for two hours with diluted antibodies against CDK9 (1:5000, Abcam, US) and glyceraldehyde-3-phosphate dehydrogenase (GAPDH) (1:2000, CST, USA). The membrane was then washed and incubated with diluted horseradish peroxidase (HRP)-labeled sheep anti-rabbit antibodies (1:1000, Beyotime, China) at $37^{\circ} \mathrm{C}$ for one hour. ECL luminescence solution was formulated and added to the front of the film, followed by five minutes of light avoidance in a dark room. The film was then placed into the imaging system for scanning.

\section{ELISA}

The levels of $H A$ and IgE in the serum were tested by mouse HA and IgE ELISA kit (Abcam, US). The concentrations of $\mathrm{HA}$ and IgE in the measured serum samples were calculated from the OD values of the samples through standard curves.

\section{Histological staining}

After the tissue specimens were fixed, they were encased in paraffin and cut into 5 - $\mu \mathrm{m}$-thick sections, and stained with hematoxylin and eosin. The pictures of the slices were taken using a Lycra microscope (magnification 200).

\section{Immunofluorescence staining}

After fixation, embedding, and sectioning of the tissue specimens, antigen repair (hyperbaric repair) was performed. The diluted antibody against CDK9 (1:100) was then added, followed by the diluted fluorescent secondary antibody (1:200).

\section{Statistical analysis}

All statistical analyses were performed by SPSS version 20 (IBM Corp, Armonk, NY). The data are presented as mean \pm standard error. Statistical significance was determined by one-way ANOVA. $\mathrm{P}<0.05$ was considered statistically significant.

\section{Results}

\section{Effect of agomiR-224 on the symptoms of AR}

After the last nasal challenge by OVA, the following nasal symptoms were observed in the mice (Figure 2):

The mice in the AR group and AR+NC-miRNA control group showed obvious allergic symptoms such as sneezing and nasal rubbing $(\mathrm{p}<0.001)$. After OVA challenge, compared to the AR group, mice in AR+agomiR224 intervention group showed a significant reduction in the numbers of nasal rubbing $(p<0.05)$ and sneezing $(p<0.05)$; There was no significant difference between AR+NC-miRNA control group and AR group, whereas the saline control group showed almost no symptoms of sneezing and nasal rubbing.

\section{Expression of miR-224 in nasal mucosa}

Similar to previous studies, ${ }^{3}$ the expression of miR-224 is significantly decreased in the nasal mucosa of AR mice. To further reveal the role of miR-224 in AR, we spotted agomiR224 into the nasal cavity of AR mice. The MiR-224 expression was significantly restored in the nasal mucosa of agomiR224-interfered AR mice, indicating successful gene transduction in these mice (Figure 3).

\section{Expressions of cytokines in the nasal mucosa and levels of IgE, HA in the serum of mice}

The involvement of multiple immunoreactive cells and cytokines in the pathogenesis of AR led us to investigate whether the upregulation of miR-224 reduces the level of IL-4 (reflecting Th2 cell levels ${ }^{13}$ ), IL-6 (reflecting the acute phase of the disease ${ }^{14}$ ), and IL-18 (reflecting the AR sustained state $^{2}$ ). The results showed that the levels of these cytokines are significantly reduced in the nasal mucosa of
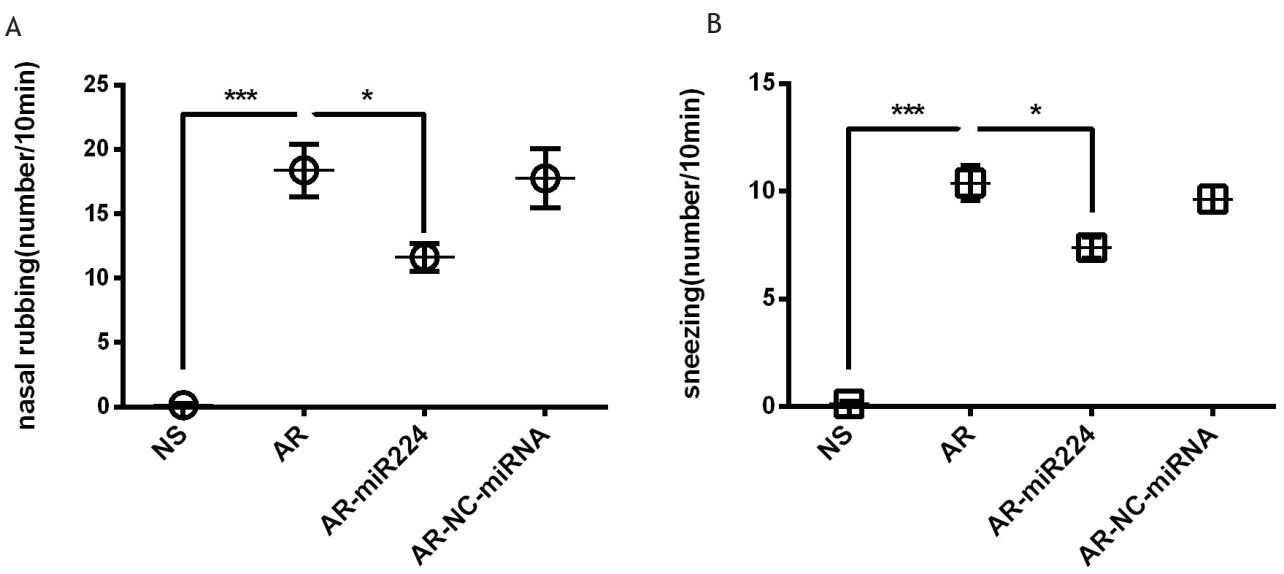

Figure 2 Nasal symptoms of mice within 10 minutes after nasal OVA challenge. (A) Count of nasal rubbing. (B) Count of sneezing. NS: saline control group, AR: AR group, AR-miR224: AR+agomiR224 intervention group, AR-NC-miRNA: AR+NC-miRNA (negative control miRNA agomir) control group. ${ }^{*} \mathrm{p}<0.05 ;{ }^{* * *} \mathrm{p}<0.001$. 


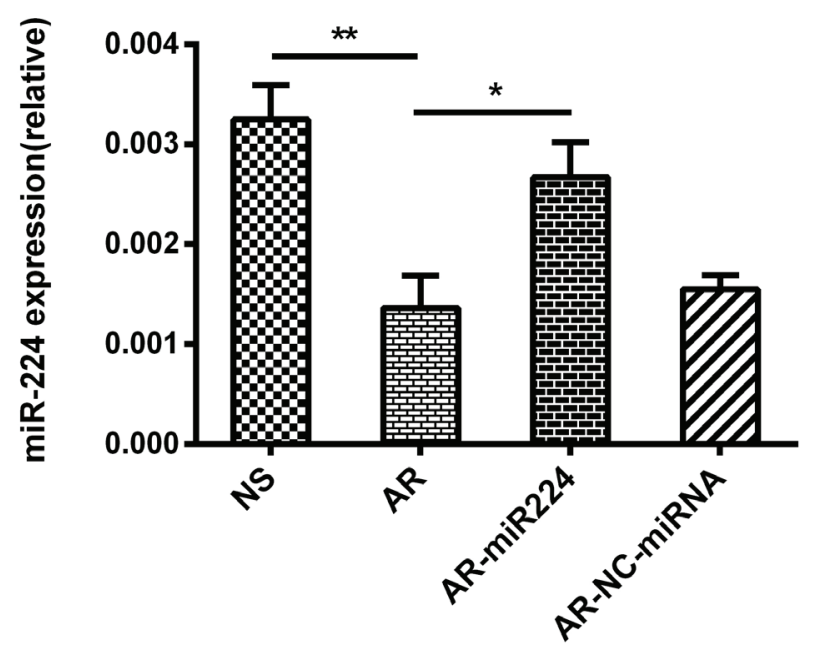

Figure 3 MiR-224 expression in nasal mucosa of mice by rt-PCR.

NS: saline control group, AR: AR group, AR-miR224: AR+agomiR224 intervention group, AR-NC-miRNA: AR+NCmiRNA (negative control miRNA agomir) control group. ${ }^{*} \mathrm{p}<0.05 ;{ }^{* *} \mathrm{p}<0.01$.

AR mice with agomiR224 intervention, compared to the AR group (Figure $4 \mathrm{~A}-\mathrm{C}$ ). The serum levels of IgE and HA were significantly up-regulated in the AR group of mice compared to the saline control group, while the levels of both significantly reduced in the agomiR224 intervention group (Figure 4D-E).

\section{CDK9 was the target gene of miR-224}

CDK9 was selected as one of the miR-224 target genes based on miRNA target gene prediction software and databases (the mating sites are shown in Figure $5 \mathrm{~A}$ ). The 7-bp fragment of the 3'UTR of the CDK9 gene is complementary to the miR-224 seed sequence (Figure 5A). As shown in Figure 5C, treatment with agomiR224 significantly reduced the luciferase activity of the 3'UTR of the wildtype pGL3-Promoter-wtCDK9 vector $(p<0.01)$, while there was no significant difference in the luciferase activity of the mutant. It was verified that CDK9 is a miR-224 target gene, and is down-regulated by miR-224.

\section{Expression and distribution of CDK9 in the nasal mucosa of mice}

We further investigated whether miR-224 affected CDK9 expression in AR. The results showed that CDK9 expression in the nasal mucosa of AR mice was significantly higher than that of normal control mice, while intra-nasal dosing of agomiR224 significantly attenuated the increase of expression of CDK9 induced by OVA sensitization (Figure 6A-B). We also investigated the expression of CDK9 in the nasal mucosa of mice of different groups by immunofluorescence. The results showed that in the AR group and the AR-NC-miRNA group, CDK9 was mainly distributed on the mucosal surface, mucosal epithelium, and glandular cells. After the agomiR224 intervention, the expression of CDK9 showed a decrease in all parts of the nasal mucosa of mice, especially in the mucosal epithelial mesenchyme. In saline normal controls, CDK9 expression was relatively low at all mucosal sites (Figure 6C).

\section{Inflammatory infiltration of the nasal mucosa}

Mouse nasal mucosal tissue was sectioned and HE stained. On further microscopic examination, a large number of inflammatory cells, including mast cells, macrophages, and eosinophils were observed under the nasal mucosa in the AR group and the AR-NC-miRNA group. Abundant vacuolated vesicles between columnar epithelial cells of the nasal mucosa were also observed, suggesting a hyperproduction of glands. In the AR-miR224 group and the NS group, the number of inflammatory cells was significantly lower and the columnar epithelial cells were more closely arranged (Figure 7).

\section{Discussion}

As a kind of refractory and recurrent disease, AR seriously affects the physical and mental health as well as the quality of life of patients. ${ }^{15,16}$ MiRNAs have immunomodulatory effects and participate in the differentiation and maturation of $\mathrm{T}$ and $\mathrm{B}$ lymphocytes, the regulation of immune response, the secretion of inflammatory cytokines, and other immune processes. ${ }^{17}$ Over the past two decades, miRNAs have emerged as key regulators that may be involved in the pathogenesis of various diseases, including in respiratory allergic diseases such as allergic rhinitis, allergic asthma, ${ }^{18,19}$ etc. It has been shown that miRNAs can down-regulate the expression of homologous target genes by cleavage of the target gene transcriptome or repression of its translation. ${ }^{20}$ Since miRNAs are more stable than mRNAs and even some proteins, researchers believe that this stability is more conducive to the regulation of gene expression in clinical diseases, including AR. ${ }^{19,21}$ For example, miR-199-3p targets Dnmt3a, resulting in hypermethylation of promoter as well as overexpression of signal transducer and activator of transcription 3(STAT3), ultimately leading to AR symptoms. ${ }^{22}$ The anti-inflammatory effect of miR-146a on AR may act by inhibiting the toll-like receptor4 (TLR4)/TNF receptor-associated factor 6 (TRAF6)/nuclear factor kappa-B (NF- $\mathrm{KB}$ ) signaling pathway. ${ }^{23}$ Matrilin 2(MATN2) can be directly targeted by miR-202-5p, and can reverse miR-202-5p-mediated $M 2$ phenotypic polarization in AR. ${ }^{24}$ Intranasal administration of lentiviral miR$135 \mathrm{a}$ in mice modulates mast cell and allergen-induced inflammation by targeting GATA-binding protein 3(GATA3). ${ }^{25}$ The CDK9-related pathway is a key factor in the regulation of mammalian gene expression, which controls several physiological processes such as cell growth, proliferation, survival, and differentiation. ${ }^{26}$ Researchers found that the inhibition of CDK9 helped to reduce cytokine production against bacterial infections, such as reducing the expression of both IL-6 and Tumor Necrosis Factor $\alpha$ (TNF- $\alpha$ ). Their 

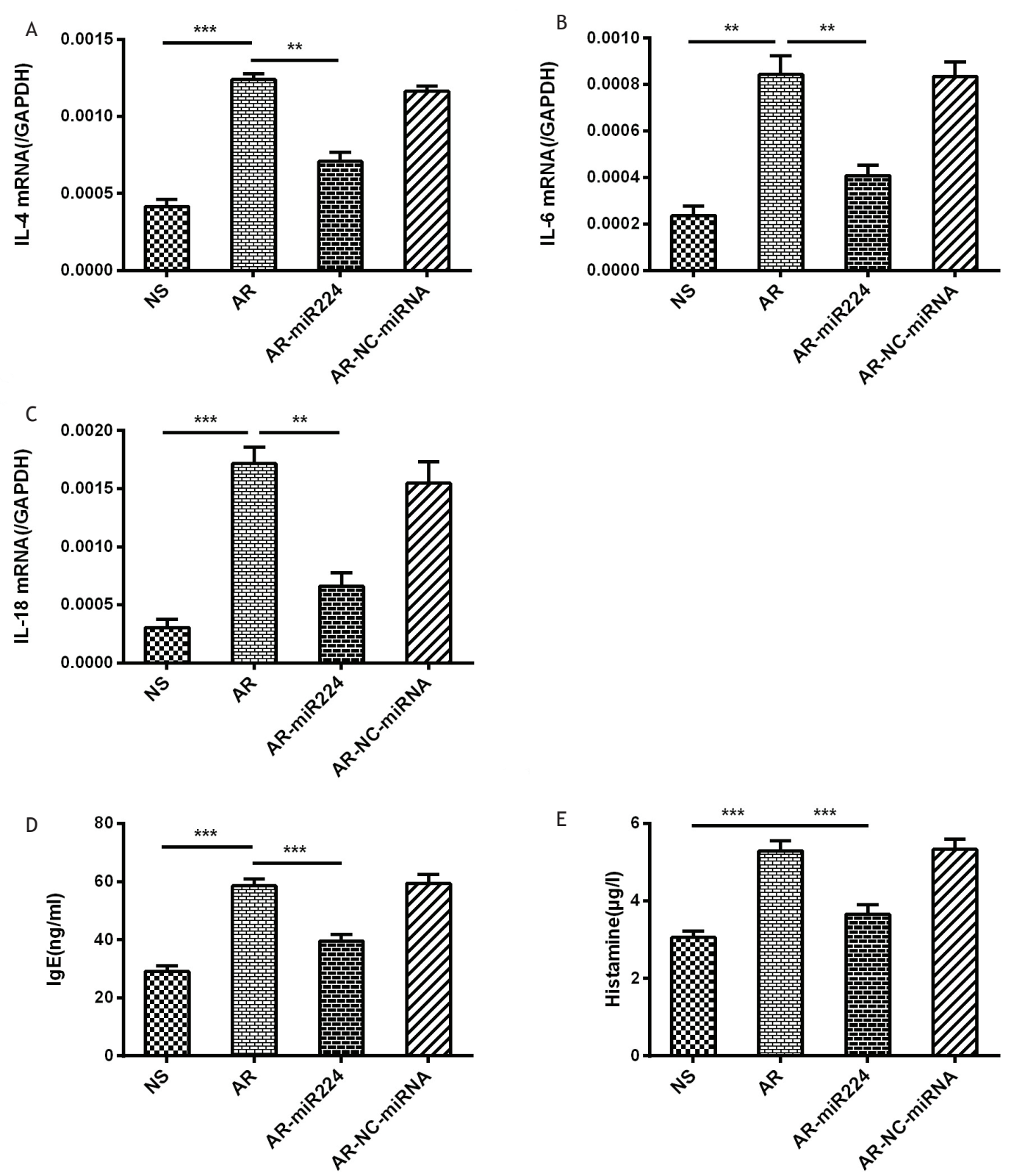

Figure 4 Expression of cytokines in the nasal mucosa of mice and levels of IgE, HA in the serum of mice. (A)-(C): Rt-PCR was used to analyze the expressions of IL-4, IL-6 and IL-18. (D)-(E): The concentrations of IgE and HA were measured by ELISA.

NS: saline control group, AR: AR group, AR-miR224: AR+agomiR224 intervention group, AR-NC-miRNA: AR+NC-miRNA (negative control miRNA agomir) control group. ${ }^{* *} \mathrm{p}<0.01 ;{ }^{* * *} \mathrm{p}<0.001$.

study also confirmed that CDK9 is involved in the innate immune response. ${ }^{4}$ In the present study, we explored for the first time, the effect of miR-224 on AR-related allergic reactions induced by OVA in a mouse model. We found that miR-224 ameliorated allergic inflammation in AR mice by targeting CDK9 and inhibiting its expression. These results provide a link between miR-224 and CDK9 in the pathogenesis of AR. Consistent with previous studies, ${ }^{3}$ we found reduced expression of miR-224 in the nasal mucosa of $A R$ model mice, further suggesting that miR-224 may negatively regulate the allergic response of AR. To avoid complications that may be caused by intravenous infusion on mice, we took the nasal drop of agomiR224 and studied the direct effect of miR-224 on nasal mucosa. As expected, miR-224 expression was significantly restored after the nasal drip with agomiR224; moreover, nasal rubbing and sneezing symptoms, concentrations of IgE and HA in serum were significantly reduced in mice in the agomiR224 intervention group compared to the AR group. At the same time, the histological analysis also revealed that columnar epithelial cells in the nasal mucosa of AR mice intervened with agomiR224 were closely aligned, and the number of inflammatory cells was significantly reduced. This suggested that agomiR224 actually relieved allergic symptoms in AR mice. 
A Conserved

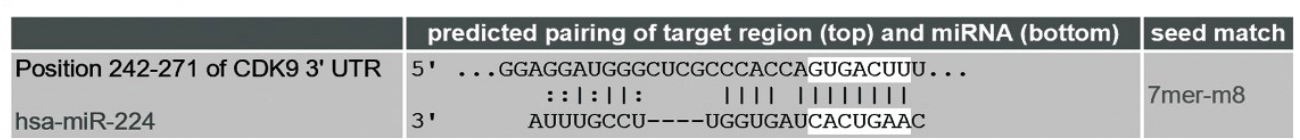

B

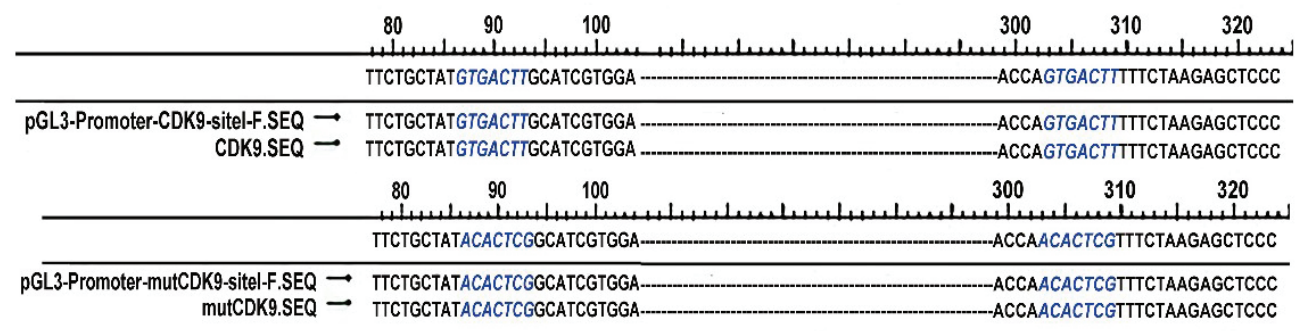

C

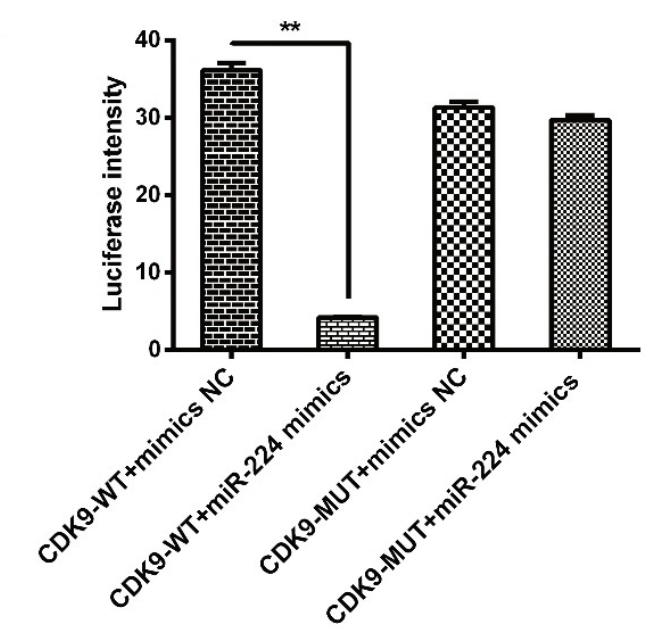

Figure 5 CDK9 was the target gene of miR-224. (A) Bioinformatics analysis: the complementary paired loci of the 3'UTR of target gene CDK9 with miR-224. (B) Partial result of CDK9-3'UTR wild-type and mutant-type recombinant vectors. The blue part shows that the miR-224 seed sequence target GTGACTT was successfully mutated to ACACTCG. (C) Result of dual luciferase activity assay. ${ }^{* *} \mathrm{p}<0.01$.

Moreover, AR is an inflammatory disease of the upper respiratory tract characterized by IgE-mediated inflammatory response, closely associated with Th2 cells ${ }^{27}$ and cytokines such as IL-4, IL-5, IL-6, IL-13, and IL18. Here, we found that upregulation of miR-224 reduced the serum levels of IL-4, IL-6, and IL-18 in AR mice, suggesting that inhibition of Th2 cytokines may underlie the anti-inflammatory effects of miR-224 on AR. In this study, our data showed that miR224 inhibited CDK9 expression through direct binding of the 3'UTR to CDK9. In the nasal mucosa of AR mice, CDK9 was mainly distributed on the mucosal surface, mucosal epithelium, and glandular cells, and its expression was up-regulated. After the intervention of agomiR224, the expression of CDK9 in all parts of the nasal mucosa of mice showed a decrease, especially in the mucosal epithelial mesenchyme. We also analyzed the nasal mucosa of patients with allergic rhinitis (without nasal polyp) as well as non-allergic structural rhinitis (results were not shown). The nasal mucosa was obtained after nasal cavity ventilation expansion, and rt-PCR revealed that miR-224 levels were decreased and CDK9 levels were increased in the nasal mucosa of patients with allergic rhinitis compared to patients with non-allergic structural rhinitis.
In conclusion, our present study demonstrated that upregulation of miR-224 effectively ameliorated pathological changes in AR by inhibiting the inflammatory response, including reducing IgE and histamine concentrations, inflammatory cell infiltration, and cytokine levels. Notably, we demonstrated that miR-224 directly targeted CDK9 and inhibited its expression. The underlying mechanisms of its specific signaling pathway need to be studied in depth. Moreover, we reported a novel approach to treat allergic rhinitis by agomiRNA interventions, in which synthetic miR224 agomir nasal drops can reduce the symptoms of AR by reducing the local allergic inflammatory response in the nasal mucosa through inhibition of the target gene CDK9. This approach might become a potential gene therapy for nasal diseases in the future.

\section{Acknowledgements}

This paper is supported by Songjiang District Science and Technology Project (19SJKJGG25); Clinical Research Plan of SHDC (16CR2029B). 


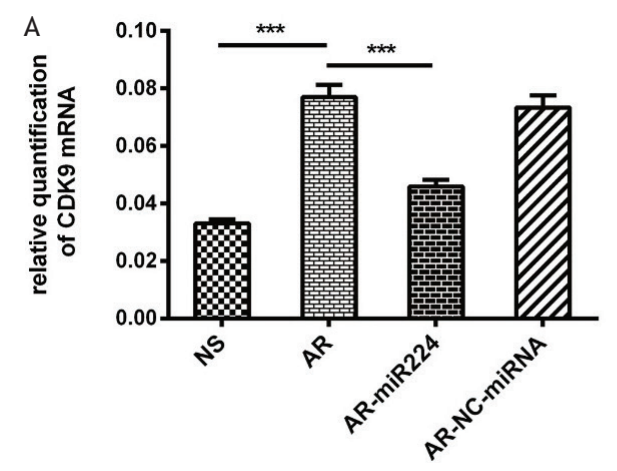

B
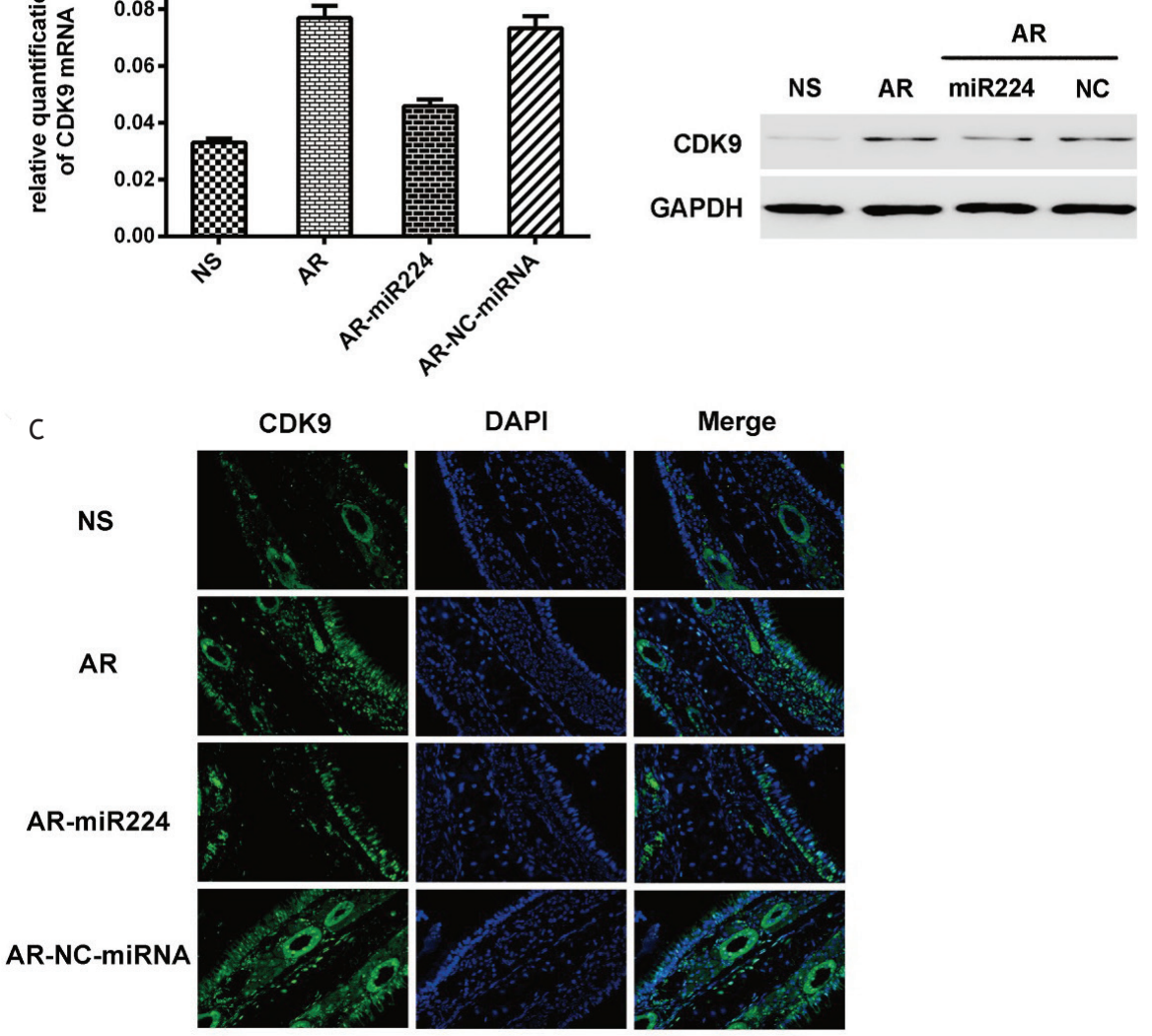

Figure 6 Expression and distribution of CDK9 in nasal mucosa of mice. (A) The level of CDK9 mRNA analyzed by Rt-PCR. (B) The CDK9 protein expression analyzed by western blotting. (C) The expression and distribution of CDK9 in the nasal mucosa of mice after immunofluorescence staining.

NS: saline control group, AR: AR group, AR-miR224: AR+agomiR224 intervention group, AR-NC-miRNA: AR+NC-miRNA (negative control miRNA agomir) control group. ${ }^{* * *} \mathrm{p}<0.001$.

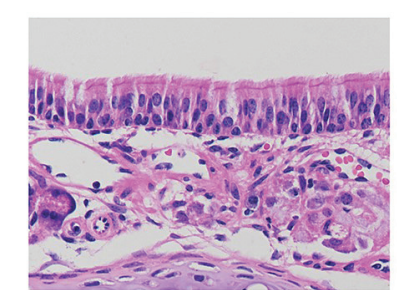

NS

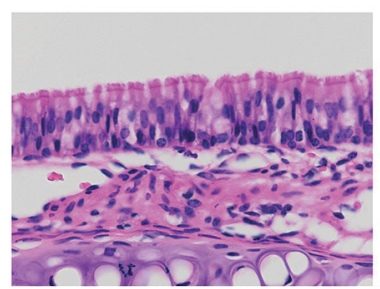

AR-miR224

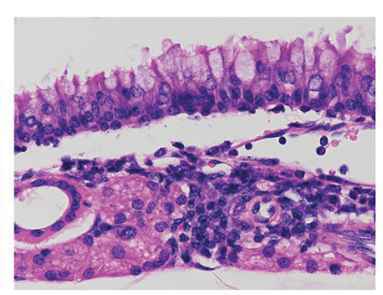

AR

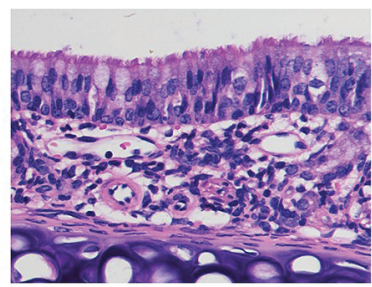

AR-NC-miRNA

Figure 7 HE staining of nasal mucosa of mice.

NS: saline control group, AR: AR group, AR-miR224: AR+agomiR224 intervention group, AR-NC-miRNA: AR+NCmiRNA (negative control miRNA agomir) control group.

\section{Potential conflict of interest}

None disclosed.

\section{References}

1. Cheng L, Chen J, Fu Q, He S, Li H, Liu Z, et al. Chinese society of allergy guidelines for diagnosis and treatment of allergic rhinitis. Allergy Asthma Immunol Res. 2018;10(4):300-353. https://doi.org/10.4168/aair.2018.10.4.300

2. Verhaeghe B, Gevaert P, Holtappels G, Lukat KF, Lange B, Van Cauwenberge $\mathrm{P}$, et al. Up-regulation of IL-18 in allergic rhinitis. Allergy. 2002;57(9):825-830. https://doi.org/10. 1034/j.1398-9995.2002.23539.x

3. Shaoqing Y, Ruxin Z, Guojun L, Zhiqiang Y, Hua H, Shudong Y, et al. Microarray analysis of differentially expressed microRNAs in allergic rhinitis. Am J Rhinol Allergy. 2011;25(6):e242 e246. https://doi.org/10.2500/ajra.2011.25.3682

4. Li J, Shi J, Pan Y, Zhao Y, Yan F, Li H, et al. Transcription modulation by CDK9 regulates inflammatory genes and RIPK3-MLKLmediated necroptosis in periodontitis progression. Sci Rep. 2019;9(1):17369. https://doi.org/10.1038/s41598-019-53910-y

5. Zhang J, Li G, Ye X. Cyclin T1/CDK9 interacts with influenza A virus polymerase and facilitates its association with cellular 
RNA polymerase II. J Virol. 2010;84(24):12619-12627. https:// doi.org/10.1128/JVI.01696-10

6. Lu Y, Tang L, Zhang Q, Zhang Z, Wei W. MicroRNA-613 inhibits the progression of gastric cancer by targeting CDK9. Artif Cells Nanomed Biotechnol. 2018;46(5):980-984. https://doi. org/10.1080/21691401.2017.1351983

7. De Falco G, Neri LM, De Falco M, Bellan C, Yu Z, De Luca A, et al. Cdk9, a member of the cdc2-like family of kinases, binds to gp130, the receptor of the IL-6 family of cytokines. Oncogene. 2002;21(49):7464-7470. https://doi.org/10.1038/sj.onc.1205967

8. Datta A, Moitra S, Das P, Mondal S, Omar Faruk S, Hazra I, et al. Allergen immunotherapy modulates sensitivity of Treg cells to apoptosis in a rat model of allergic asthma. 2017;9(15):1239-1251. https://doi.org/10.2217/imt-2017-0038

9. Hellvard A, Zeitlmann L, Heiser U, Kehlen A, Niestroj A, Demuth HU, et al. Inhibition of CDK9 as a therapeutic strategy for inflammatory arthritis. Sci Rep. 2016;6:31441. https://doi. org/10.1038/srep31441

10. Schmerwitz UK, Sass G, Khandoga AG, Joore J, Mayer BA, Berberich $\mathrm{N}$, et al. Flavopiridol protects against inflammation by attenuating leukocyte-endothelial interaction via inhibition of cyclin-dependent kinase 9. Arterioscler Thromb Vasc Biol. 2011;31(2):280-288. https://doi.org/10.1161/ATVBAHA. 110.213934

11. Sonawane YA, Taylor MA, Napoleon JV, Rana S, Contreras JI, Natarajan A. Cyclin dependent kinase 9 inhibitors for cancer therapy. J Med Chem. 2016;59(19):8667-8684. https://doi. org/10.1021/acs.jmedchem.6b00150

12. Hu H, Ramezanpour M, Hayes AJ, Liu S, Psaltis AJ, Wormald PJ, et al. Sub-inhibitory clindamycin and azithromycin reduce $S$. aureus exoprotein induced toxicity, inflammation, barrier disruption and invasion. J Clin Med. 2019;8(10):1617. https://doi. org $/ 10.3390 / j \mathrm{jm} 8101617$

13. Ashraf MI, Shahzad M, Shabbir A. Oxyresveratrol ameliorates allergic airway inflammation via attenuation of IL-4, IL-5, and IL-13 expression levels. Cytokine. 2015;76(2):375-381. https:// doi.org/10.1016/j.cyto.2015.09.013

14. Tanaka T, Narazaki M, Kishimoto T. IL-6 in inflammation, immunity, and disease. Cold Spring Harb Perspect Biol. 2014; 6(10): a016295. https://doi.org/10.1101/cshperspect. a016295

15. Ferrante G, Montalbano L, Cilluffo G, Malizia V, Marchese D, La Grutta S. Beclomethasone dipropionate hydrofluoroalkane for the treatment of allergic rhinitis. Expert Rev Clin Immunol. 2016;12(3):279-288. https://doi.org/10.1586/17446 66X.2016.1118347
16. Shi Q, Lei Z, Cheng G, Li D, Wang Q, Luo S, et al. Mitochondrial ROS activate interleukin-1beta expression in allergic rhinitis. Oncol Lett. 2018;16(3):3193-3200. https://doi.org/10.3892/ ol.2018.8984

17. Liang T-J, Qin C-Y. The emerging role of microRNAs in immune cell development and differentiation. APMIS. 2009;117(9):635643. https://doi.org/10.1111/j.1600-0463.2009.02520.x

18. Panganiban RP, Wang Y, Howrylak J, Chinchilli VM, Craig TJ, August A, et al. Circulating microRNAs as biomarkers in patients with allergic rhinitis and asthma. J Allergy Clin Immunol. 2016;137(5):1423-1432. https://doi.org/10.1016/j. jaci.2016.01.029

19. Zhang XH, Zhang YN, Liu Z. MicroRNA in chronic rhinosinusitis and allergic rhinitis. Curr Allergy Asthma Rep. 2014;14(2):415. https://doi.org/10.1007/s11882-013-0415-3

20. Bartel DP. MicroRNAs: genomics, biogenesis, mechanism, and function. Cell. 2004;116(2):281-297. https://doi.org/10.1016/ S0092-8674(04)00045-5

21. Teng $\mathrm{Y}$, Zhang $\mathrm{R}$, Yu H, Wang $\mathrm{H}$, Hong $\mathrm{Z}$, Zhuang $\mathrm{W}$, et al. Altered microRNA expression profiles in activated mast cells following IgE-Fc\&RI cross-linking with antigen. Cell Physiol Biochem. 2015;35(6):2098-2110. https://doi.org/10. 1159/000374016

22. Cui X, Guo Y, Wang Q, Li X. MiR-199-3p-Dnmt3a-STAT3 signalling pathway in ovalbumin-induced allergic rhinitis. Exp Physiol. 2019;104(8):1286-1295. https://doi.org/10.1113/EP087751

23. Wang J, Cui Z, Liu L, Zhang S, Zhang Y, Zhang Y, et al. MiR-146a mimic attenuates murine allergic rhinitis by downregulating TLR4/TRAF6/NF-KB pathway. Immunotherapy. 2019; 11(13): 1095-1105. https://doi.org/10.2217/imt-2019-0047

24. Wang L, Liu X, Song X, Dong L, Liu D. MiR-202-5p promotes M2 polarization in allergic rhinitis by targeting MATN2. Int Arch Allergy Immunol. 2019;178(2):119-127. https://doi.org/10. 1159/000493803

25. Deng $\mathrm{Y}-\mathrm{Q}$, Yang $\mathrm{Y}-\mathrm{Q}$, Wang $\mathrm{S}-\mathrm{B}$, Li F, Liu $\mathrm{M}-\mathrm{Z}$, Hua Q-Q, et al. Intranasal administration of lentiviral miR-135a regulates mast cell and allergen-induced inflammation by targeting GATA-3. PLoS One. 2015;10(9):e0139322. https://doi.org/10.1371/journal.pone.0139322

26. Romano G, Giordano A. Role of the cyclin-dependent kinase 9-related pathway in mammalian gene expression and human diseases. Cell Cycle. 2008;7(23):3664-3668. https://doi. org/10.4161/cc.7.23.7122

27. Ellis AK, Tenn MW. Advances in rhinitis: models and mechanisms. Ann Allergy Asthma Immunol. 2018;121(1):61-64. https://doi.org/10.1016/j.anai.2017.10.008 